\title{
Relation Entre Le Somatotype Et La Qualite De Vitesse Chez Des Jeunes De Cote d'Ivoire
}

\author{
Siaka Coulibaly, (Doctorant) \\ Laboratoire de Biomorphologie et d'Anthropologie physique, Département \\ de Paléoanthropologie, Institut des sciences Anthropologiques de \\ Développement, Université Félix Houphet Boigny, Abidjan, Côte d'Ivoire \\ N'guessan Kouamé, (Maître-Assistant) \\ Institut National de la Jeunesse et des Sports, Abidjan, Côte d'Ivoire \\ Firmin Kouakou Kouassi (Maître-Assistant) \\ Jérôme Kouakou Kouadio, (Maître-Assistant) \\ Jean-Bertin Beugré, (Maître de Conférences) \\ Laboratoire de Biomorphologie et d'Anthropologie physique, Département \\ de Paléoanthropologie, Institut des sciences Anthropologiques de \\ Développement, Université Félix Houphet Boigny, Abidjan, Côte d'Ivoire \\ Jean-Claude Pineau, (Directeur de Recherches) \\ CNRS UPR 2147, Dynamique de l'évolution humaine, Paris, France
}

doi: 10.19044/esj.2016.v12n23p378 URL:http://dx.doi.org/10.19044/esj.2016.v12n23p378

\begin{abstract}
The aim of this study is to establish a link between the performance at speed race and the somatotype within young students from Côte d'Ivoire who are candidates for the recruitment test organized by the National Institute of Youth and Sports. The study was performed on 669 girls at $21.80+2.26$ year old and 1859 boys at $22.11+2.26$ years. Anthropometrical measures were made out in order to estimate somatotype. For the speed, the subjects underwent a $80 \mathrm{~m}$ speed race test for girls and a $100 \mathrm{~m}$ speed race for boys. After a descriptive analysis of the somatotype components and the performance of girls and boys, we made a distribution of the gaps in performance (estimated performance - real performance) estimated by the regression and the real performance in order to check normality. To examine the relationship between somatotype, cormic index and the speed race, an equation of multiple regressions was performed. The distribution of the gaps in performance (estimated performance - real performance) estimated by the regression and real performance is normally distributed both in girls and boys. The results also indicate that a multiple and statistically significant link $(p<0.05)$ exists between the performance and three (3) variables:
\end{abstract}


endormorphy, ectomorphy and cormic index with boys and two (2) components of somatotype: endomorphy, mesomorphy with girls. The present study has allowed establishing a relationship between the performance at speed race and some of the components of the somatotype. The performance at $100 \mathrm{~m}$ for boys and $80 \mathrm{~m}$ for girls is well the result of a specific morphotype (endomorph) limiting the realization of good performances by these sprinters.

Keywords: Somatotype, speed, morphotype, sprinters

\section{Resume}

L’objectif de cette étude est d'établir un lien entre la performance à la course de vitesse et le somatotype chez des jeunes élèves ivoiriens candidats au test d'entrée à l'Institut National des Sports. Cette étude a porté sur 669

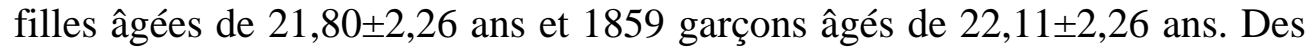
mesures anthropométriques ont été effectuées pour déterminer le somatotype. Pour la vitesse, les sujets ont été soumis à un test de course de vitesse sur 80 mètres pour les filles et sur 100 mètres pour les garçons. Après une analyse descriptive des composants du somatotype et de la performance chez les filles et chez les garçons, nous avons fait une distribution des écarts de performance (perf estimée - perf réelle) estimée par la régression et la performance réelle pour vérifier la normalité. Pour examiner la relation entre le somatotype et la course de vitesse, une équation de régression multiple a été établie. La distribution des écarts de performance (perf estimée - perf réelle) estimée par la régression et la performance réelle est gaussienne aussi bien chez les filles que chez les garçons. Les résultats indiquent également qu'il existe une liaison multiple et statistiquement significative à $\mathrm{p}<0.05$ entre la performance à la course de vitesse et 3 variables: endomorphie, ectomorphie et Indice cormique chez les garçons et deux composantes du somatotype: endomorphie, mésomorphie chez les filles. La présente étude a permis d'établir une relation entre la performance à la course de vitesse et des composants du somatotype. La performance au $100 \mathrm{~m}$ pour les garçons et $80 \mathrm{~m}$ pour les filles est bien la résultante d'un morphotype spécifique (endomorphe) limitant la réalisation de bonnes performance chez ces sprinters.

Mots Clés : Somatotype, Vitesse, Morphotype, Sprinters

\section{Introduction}

L'entraînement sportif semble provoquer chez le jeune sportif, des modifications physiques et physiologiques immédiates ou à long terme. Le niveau et la nature de ces différentes variations peuvent être appréciés chez 
l'individu à travers certains tests de qualités physiques, notamment l'endurance, la souplesse, la force, la vitesse, etc. La réussite à ceux-ci renvoie à ce que l'on appelle la performance sportive. Certains s'adonnent aux activités physiques et sportives soit, pour conserver leur équilibre physique, soit pour corriger des faiblesses biologiques et physiologiques. D'autres, en revanche, font du sport, une activité régulière, c'est-à-dire un métier, une profession dont les processus d'apprentissage et d'acquisition pourraient se réaliser dans une sorte de concurrence, de compétition. De telles confrontations interindividuelles sont souvent mesurées ou évaluées. C'est le cas de l'athlétisme, notamment la course de vitesse.

La performance sportive se réfère aux possibilités physiques maximales d'un individu dans une discipline sportive (Platonov, 1984). Elle semble impliquer ainsi les acquisitions physiques et morphologiques du sportif, notamment son somatotype. En effet, la vitesse maximale de la bipédie humaine semble s'organiser autour de gradients biométriques, qui en optimisent l'efficacité (Sedeaud et al., 2014). L'on pourrait ainsi observer l'influence de la structure corporelle dans plusieurs disciplines, notamment la course de fond. Ici, l'athlète disposant des grands membres inférieurs serait plus susceptible de performance de qualité (Mimouni et Antipov, 1986). De même, Smirniotou et al. (2008) ont montré que la performance au 100m est fortement associée à des paramètres de puissance. La détermination de somatotype représente ainsi un point de départ important pour choisir le sport le mieux adapté à chaque sujet (Ripari et al., 2008). Elle revêt une grande importance pour l'anthropologie médicale et physique ainsi que la science du sport (Yang et al., 2016). La présente étude est une contribution à la détection, à l'orientation et à l'amélioration de la performance à la course de vitesse par la prise en compte du somatotype. Elle porte sur des candidats au test d'entrée à l'Ecole Normale Supérieure de l'Education Physique et des Sports (ENSEPS) au sein de l'Institut National de Jeunesse et des Sports (INJS). Cependant, la performance à la vitesse peut aussi être la résultante de multiples critères d'ordre morphologique mais également des critères généraux, notamment l'âge. L'objectif de cette étude est d'établir un lien entre la performance au sprint et le somatotype chez des jeunes élèves ivoiriens candidats au test d'entrée à l'INJS.

\section{Materiels et methodes \\ Materiels}

L'examen de la vitesse par la prise en compte du somatotype a porté sur les candidats au test d'entrée à l'ENSEPS au sein de l'INJS. Les sujets ont été informés de l'étude et ont tous donné leur consentement. Pour les moins de 18 ans, les parents ont fourni un document d'autorisation. 
Ont été retenus dans cette étude les candidats déclarés aptes à la visite médicale, c'est-à-dire ne présentant aucune contre-indication à la pratique du sport. En référence à ces critères susmentionnés, 2528 jeunes (669 filles et 1859 garçons) ont été retenus sur les 3156 candidats qui se sont présentés pour le test.

\section{Methodes}

Il s'agit d'une étude observationnelle transversale et analytique. Elle a été réalisée à travers un recueil de mesures anthropométriques, notamment le poids $(\mathrm{kg})$, la stature $(\mathrm{cm})$, la taille assis, les circonférences du bras relâché et du mollet, les diamètres bicondilaires de l'humérus et du fémur ainsi que la somme de quatre (4) plis cutanés (tricipital, sous-scapulaire, supra-iliaque et sural). Ces données ont servis au calcul du somatotype selon la méthode anthropométrique de Heath et Carter (1990) énoncée comme suit :

Endomorphie $=-0,7182+0,1451 X-0,00068 X^{2}+0,0000014 X^{3}$ où $\mathrm{X}=$ (Somme des plis cutanés triceps, subscapulaire et suprailiaque) $\times$ (170,18/taille cm)

Mésomorphie $=[(0,858 \mathrm{H})+(0,601 \mathrm{~F})+(0,188 \mathrm{CBC})+$ $(0,161 \mathrm{CPC})]-(0,131 \mathrm{E})+4,5$ où $\mathrm{H}=$ largeur de l'humérus au coude $(\mathrm{cm}) ; \mathrm{F}=$ largeur du fémur au genou $(\mathrm{cm})$; $E=$ Taille $(\mathrm{cm})$; CBC= Circonférence du bras (CB) corrigée : (Convertir le pli cutané tricipital en cm (divisé par 10), soustraire la valeur de la circonférence bicipitale pour obtenir la valeur corrigée) ; CPC= Circonférence du mollet (CP) corrigée (Convertir le pli cutané du mollet en cm (divisé par 10), soustraire la valeur de la circonférence du mollet pour obtenir la valeur corrigée)

Ectomorphie $=\left[\mathrm{E} /(\mathrm{P})^{1 / 3}\right)$ 0,732]-28,58 où $\mathrm{E}=$ taille $; \mathrm{P}=$ poids.

Il faut tenir compte que: Si 38,25< $\left[\mathrm{E} /(\mathrm{P})^{1 / 3}\right]<40,75$, l'ectomorphie $=\left(\left[\mathrm{E} /(\mathrm{P})^{1 / 3}\right]\right.$ 0,463)-14,63

Si $\left[E /(P)^{1 / 3}\right]<38,25$, l'ectomorphie $=0,1$.

D'une façon complémentaire aux composant du somatotype, nous avons pris en compte l'indice cormique donné par la formule suivante:

Indice cormique $(\mathrm{IC})=($ (Taille-assis $) /$ (Taille debout) $) \times 100$ (Larem et Pigearias, 1990)

Pour la vitesse, les sujets ont été soumis à un test de course de vitesse sur 80 mètres pour les filles et sur 100 mètres pour les garçons. Les temps mis pour parcourir ces distances ont été enregistrés par des enseignants spécialistes d'athlétisme à l'aide de chronomètres à temps multiples au centième de seconde.

Après avoir vérifié que la distribution des données retenues est normale, une équation de régression multiple entre la performance à la course de vitesse et les composants du somatotype a été établie. Tous les 
tests statistiques ont été effectués à l’aide du logiciel IBM SPSS version 22. Le seuil de significativité est fixé à 5\%.

\section{Resultats}

Nous avons regroupé dans le tableau I les valeurs moyennes des composants du somatotype, de l'indice cormique et de la performance chez les garçons et chez les filles.

Tableau I : Statistiques descriptives des composants du somatotype et la performance sportive au $100 \mathrm{~m}$ chez les garçons et au $80 \mathrm{~m}$ chez les filles

\begin{tabular}{ccc}
\hline Variables & Moyennes et Ecart-types & Médiane \\
\hline Garçons (n=1859) & & \\
Performance au 100m & $13.70 \pm 0.79$ & 13.64 \\
Endomorphie & $1.79 \pm 0.78$ & 1.64 \\
Mésomorphie & $3.24 \pm 1.24$ & 3.22 \\
Ectomorphie & $3.25 \pm 1.01$ & 3.25 \\
Indice Cormique (IC) & $50.75 \pm 1.55$ & 50.83 \\
\hline Filles (n=669) & & \\
Performance au 80m & $13.58 \pm 0.98$ & 13.50 \\
Endomorphie & $3.78 \pm 1.08$ & 3.80 \\
Mésomorphie & $3.44 \pm 1.42$ & 3.40 \\
Ectomorphie & $2.97 \pm 1.12$ & 3.00 \\
Indice Cormique (IC) & $51.86 \pm 1.61$ & 51.87 \\
\hline
\end{tabular}

La distribution des écarts entre la performance estimée par la régression et la performance réelle est gaussienne et montre que:

Chez les garçons (figure 1):

753 athlètes sur 1859 ont des écarts de performance (perf estimée perf réelle) compris entre \pm 0.5 seconde soit $40.50 \%$

551 athlètes sur 1859 ont des écarts compris entre [- $1 \mathrm{~s}$ et $-0.5 \mathrm{~s}$ ] et [0.5 s et +1 s] soit $29.63 \%$.

$40.50+29.63=70.13 \%$ des athlètes ont des écarts compris entre \pm 1 seconde. 


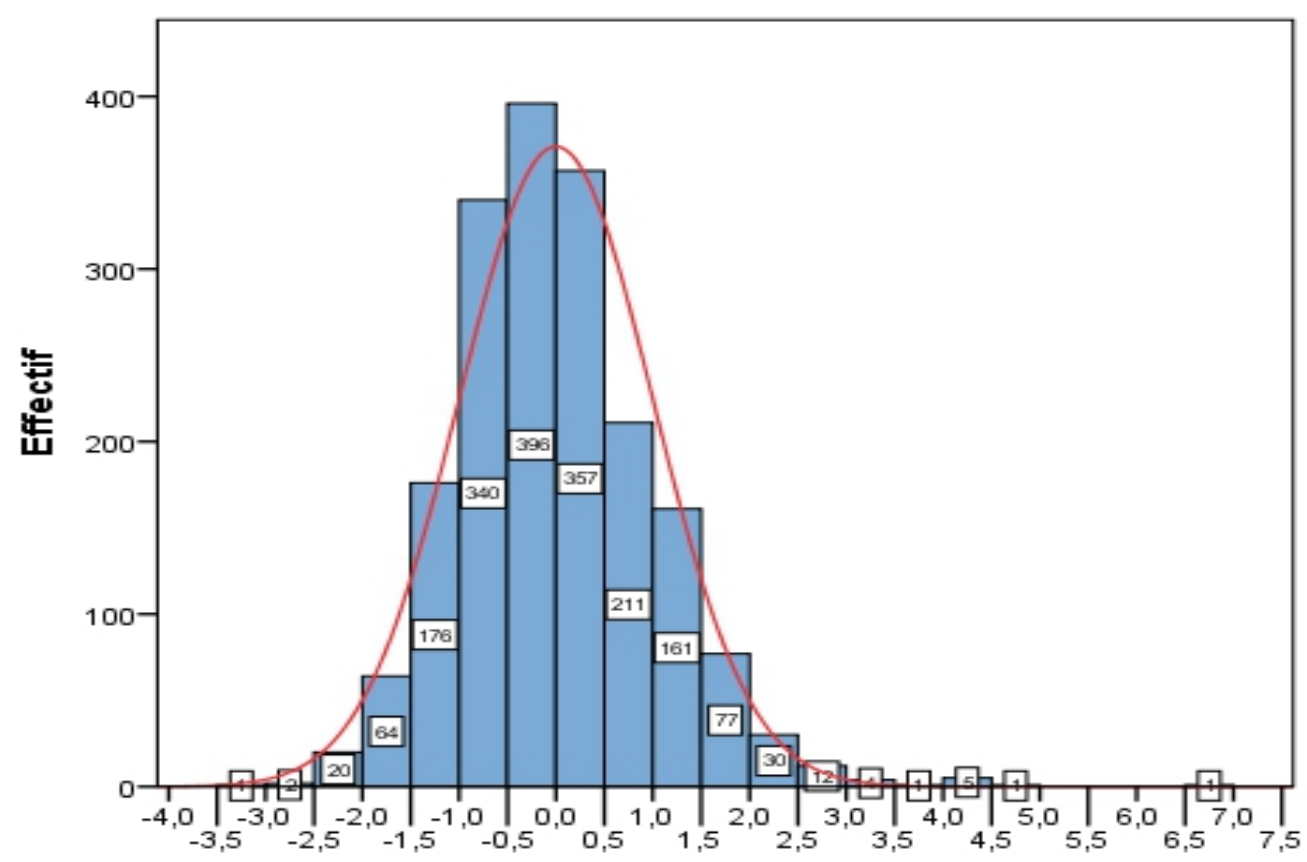

Figure 3 : Distribution des écarts de performance entre la valeur estimée par la régression et la performance réelle chez les garçons

Il existe une liaison significative (exponentielle inverse) $\quad(\mathrm{r}=-0.61)$ entre la composante ectomorphie et la composante mésomorphie. (Figure 2).

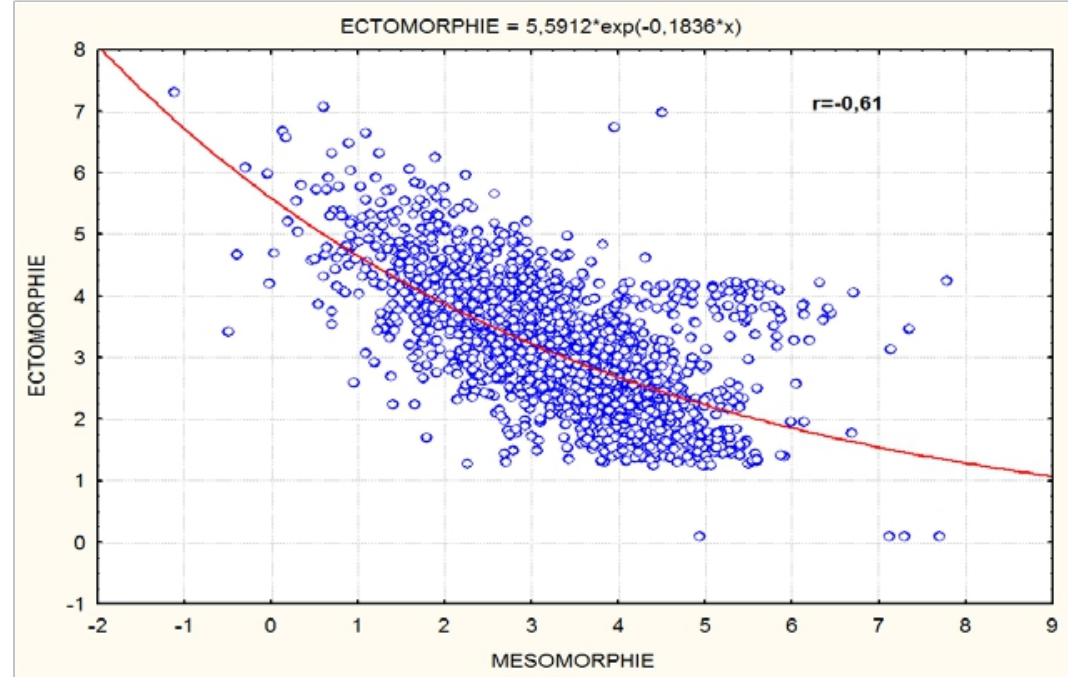

Figure 2: Relation entre les composantes ectomorphie et mésomorphie chez les garçons

\section{Chez les filles (figure 3)}

254 athlètes sur 669 ont des écarts de performance (perf estimée perf réelle) compris entre \pm 0.5 seconde soit $37.96 \%$. 
219 athlètes sur 669 ont des écarts de performance compris entre [$0.5 \mathrm{~s}$ et $-1 \mathrm{~s}]$ et $[0.5 \mathrm{~s}$ et $1 \mathrm{~s}$ ] soit $32.73 \%$.

$70.69 \%$ des athlètes ont des écarts de performance compris entre \pm 1 seconde.

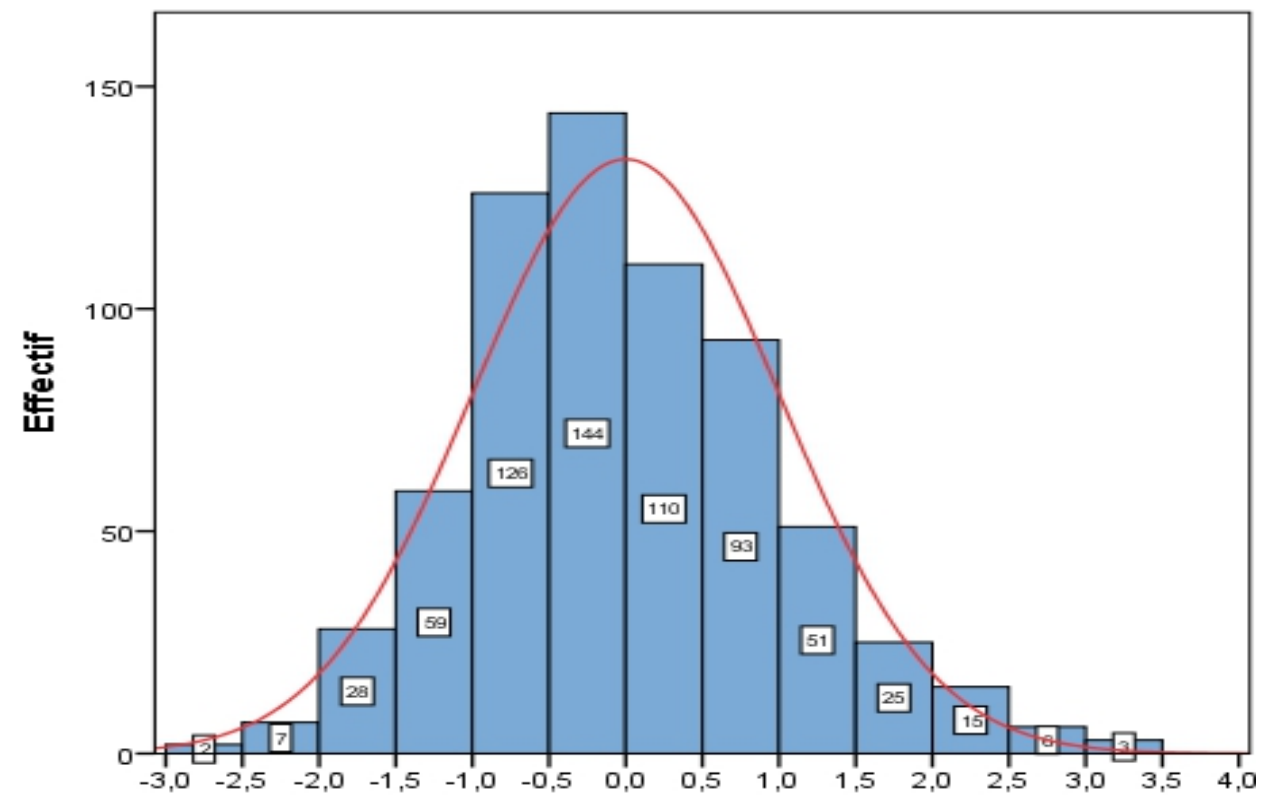

Figure 3 : Distribution des écarts de performance entre la valeur estimée par la régression et la performance réelle chez les filles.

De même, chez les filles, les valeurs moyennes des variables retenues (Tableau I) montrent que:

- Endomorphie : $\mathrm{m}=3.78 \pm 1.08$, la distribution est gaussienne car la médiane qui sépare l'effectif en deux effectifs égaux soit $50 \%$ des sujets est 3.8 très proche de la moyenne

-Mésomorphie: $\mathrm{m}=3.44 \pm 1.42$, la distribution est gaussienne car la médiane qui sépare l'effectif en deux effectifs égaux soit 50\% des sujets est 3.4 très proche de la moyenne

-Ectomorphie : $\mathrm{m}=2.97 \pm 1.12$, la distribution est gaussienne car la médiane qui sépare l'effectif en deux effectifs égaux soit 50\% des sujets est 3.0 très proche de la moyenne.

-Indice Cormique (IC) : $\mathrm{m}=51.86 \pm 1.61$, la distribution est gaussienne car la médiane qui sépare l'effectif en deux effectifs égaux soit $50 \%$ des sujets est 51,87 très proche de la moyenne.

De la même façon, la performance à la course de vitesse suit une loi normale gaussienne car $\mathrm{m}=13.58 \pm 0.98$ et la médiane soit $50 \%$ des sujets se situe à une performance de 13.5 secondes.

Une équation de régression multiple a ainsi été établie à partir d'une recherche ascendante des composants du somatotype et de l'indice cormique. 
Les résultats de l'analyse de la régression multiple sont indiqués dans le tableau II pour les garçons et pour les filles et montrent qu'il existe chez les garçons une liaison multiple entre la performance et trois variables: ectomorphie, endomorphie et l'indice Cormique et 2 composantes du somatotype: endomorphie et mésomorphie chez les filles.

Tableau II: Estimation de la performance sportive au $100 \mathrm{~m}$ pour les garçons et au $80 \mathrm{~m}$ pour les filles à partir des composants du somatotype ; test " $t$ " Student et $\mathrm{P}$ value pour chaque variable.

\begin{tabular}{ccccc}
\hline Variables & $\mathrm{A}$ & Béta & $\mathrm{t}$ & $\mathrm{p}$ \\
\hline Garçons (n=1859) & & & & \\
Constante & 13.027 & & 150.024 & 0.000 \\
Endomorphie & 0.159 & 0.159 & 6.610 & 0.000 \\
Ectomorphie & 0.121 & 0.155 & 6.472 & 0.000 \\
Indice Cormique & 0.048 & 0.094 & 3.966 & 0.000 \\
\hline Filles (n=669) & & & & \\
Constante & 12.380 & & 92.91 & 0.000 \\
Endomorphie & 0.246 & 0.270 & 6.43 & 0.000 \\
Mésomorphie & 0.078 & 0.113 & 2.68 & 0.007 \\
\hline
\end{tabular}

La régression multiple donnant une estimation de la performance en fonction des variables retenues est :

Chez les garçons: Performance $=0.142$ ectomorphie +0.155 endomorphie +0.048 Indice cormique +10.521 avec $r=0.205 \quad \mathrm{p}<0.001$. L'erreur standard d'estimation ( $\mathrm{SEE}=0.77$ secondes) avec $\mathrm{n}=1859$ athlètes.

Chez les filles: Performance $=0.247$ endomorphie +0.078 mésomorphie +12.38 avec $r=0.34 \mathrm{p}<0.01$. L'erreur standard d'estimation est ( $\mathrm{SEE}=0.93$ seconde) avec $n=669$.

\section{Discussion}

l'exigence d'une forme physique spécifique pour une bonne performance en fonction des sports a été mis en évidence dans différentes études (Adhikari et al., 2014 ; Perroni et al., 2015). Cette implication du morphotype serait plus avantageuse pour le sportif lorsqu'il s’agit de performance sportive. Des observations faites auprès des jeunes ivoiriens permettent de réexaminer cette corrélation. La régression multiple donnant une estimation de la performance en fonction des composants du somatotype montre que :

Chez les garçons il existe une liaison statistiquement significative entre la performance sportive au $100 \mathrm{~m}$ et trois variables: l'ectomorphie, l'endomorphie et l'indice cormique. Visiblement, la composante de la mésomorphie n'intervient pas dans la prédiction de la performance. L’indice cormique moyen chez ces derniers est de 50.75 \pm 1.55 . Cette valeur les classe dans la catégorie des brachicormes (buste cout et longues jambes) (Vallois, 1944 : Larem et Pigearias, 1990) les prédisposant normalement à la course à 
pied. Or, la performance moyenne obtenue par nos sujets au 100m (13.70s \pm 0.79$)$ est faible.

Cela est probablement dû au fait que ce qui différencie les sujets ce n'est pas la composante "mésomorphie" qui reste une composante "essentielle" chez les sprinters mais plutôt les deux autres composantes sachant qu'il existe de nombreuses combinaisons individuelles possibles entre ces composantes (phénomène de compensation). On sait que l'endomorphie est une composante qui traduit l'adiposité et que plus elle est élevée plus le sujet aura une performance sportive plus limitée. C'est ce que confirment les résultats concernant les garçons, la composante endomorphie influence positivement la performance sportive (temps mis). Quant à l'ectomorphie, cette composante est inversement proportionnelle ( $r=-0.61)$ à la mésomorphie. La différenciation des performances au $100 \mathrm{~m}$ est essentiellement liées aux composantes ectomorphie et endomorphie car la composante de mésomorphie bien qu'elle soit très importante dans la constitution physique (muscle et ossature) elle n'est pas discriminante au regard de la performance sportive. Dans la contribution générale de la régression multiple donnant l'estimation de la performance en fonction des 3 composantes du somatotype, la mésomorphie n'intervient pas significativement. Pour autant elle reste indispensable à la réalisation de bonne performance.

Chez les filles, les résultats montrent qu'il existe une liaison statistiquement significative entre la performance sportive au $80 \mathrm{~m}$ et les composants de l'endomorphie et de la mésomorphie. La composante de l'ectomorphie n'intervient pas car la valeur du "t" de Student n'est pas significative $(\mathrm{t}=-0.79)$ alors que la valeur de "t" pour l'endomorphie est de 6.35 et pour la mésomorphie de 2.28 (Tableau 2).

Comme chez les garçons, la composante la plus discriminante ne porte pas sur la mésomorphie qui reste la composante essentielle mais plutôt sur l'endomorphie (composante adipeuse) qui permet de différencier les performances des filles. Plus cette composante est élevée et plus la performance (temps de parcours) est élevée et par conséquent moins bonne. De la même façon que chez les garçons, les composantes du somatotype ont une contribution significative dans la réalisation de la performance chez les filles. Il apparait selon les résultats que la composante de l'endomorphie permet de différencier les performances des filles bien que cette composante joue un rôle défavorable dans l'optimisation de la performance sportive.

Ces spécificités morphologiques témoignent de certaines exigences physiques de la pratique sportive ou à une forme d'élimination naturelle qui écarte les individus dont la performance est jugée insuffisante (Léone et Lariviere, 1994). Par exemple, chez les garçons comme chez les filles, c'est la composante de l'endomorphie qui permet de différencier les performances 
à la course de vitesse bien que cette composante soit un facteur limitant dans l'optimisation de la performance sportive. Ces résultats ne sont pas en accord avec ceux obtenus par Adodo et al., 2015 et Vucetić et al. (2008). Les premiers ont identifié les composantes ectomorphie et mésomorphie comme dominant chez des jeunes athlètes nigérians. Dans la seconde étude portée sur des jeunes athlètes Croates, c'est la composante mésomorphie qui est la caractéristique dominante chez tous les groupes d'athlètes (sprinters, coureurs d'endurance, coureurs de demi-fond et coureurs de longue distance), alors que la composante ectomorphique est la moins marquée.

De même, Sedeaud et al. (op. cit.) ont mis en évidence la relation entre l'anthropométrie et la performance sur l'ensemble des distances en athlétisme. Ils ont trouvé une corrélation positive entre le poids et la taille, facteurs essentiels dans la détermination des composants du somatotype, avec la vitesse. Le poids et la taille semblent ainsi jouer un rôle important dans les processus de sélection du sport mondial. En ce sens, le morphotype pourrait apporter un regard nouveau sur ces mécanismes à l'échelle individuelle et contribuer à la détection, à l'orientation et à l'amélioration de la performance sportive notamment à la course de vitesse. Selon Ripari et al. (op. cit.), la détermination du somatotype chez les sportifs représente un point de départ important pour choisir le sport le mieux adapté à chaque sujet.

En définitive, dans cette étude, chez les garçons comme chez les filles, la composante mésomorphie ne permet pas à elle seule de différencier les performances des sprinters bien qu'elle constitue une composante essentielle. La composante la plus discriminante porte sur l'endomorphie (composante adipeuse) qui permet de différencier les performances des filles et des garçons bien que cette composante soit un facteur limitant dans l'optimisation de la performance sportive.

Ce travail est une étude transversale mettant en rapport le somatotype et la performance à la course de vitesse. Les résultats devront donc être interpréter avec un peu de prudence. La régression multiple donnant une estimation de la performance sportive en fonction des composantes du somatotype représente un faible pourcentage de la dispersion des performances sportives chez les garçons comme chez les filles. Par conséquent, cette estimation contribue significativement dans la possibilité de situer les meilleurs sprinters vis à vis des autres sprinters.

\section{Conclusion}

Dans l'analyse de la performance sportive, certains auteurs (Smirniotou et al., op. cit.) ont montré que la performance au $100 \mathrm{~m}$ est fortement associée à des paramètres de puissance. D’autres se sont intéressés aux stades de développement physique de l'individu en rapport avec la 
performance (Lowrey, 1986 ; Shephard, 1982). La présente étude a permis d'établir une étroite liaison entre la performance à la course de vitesse, les composants du somatotype et l'indice cormique. En définitive cette étude montre que la performance au $100 \mathrm{~m}$ pour les garçons et $80 \mathrm{~m}$ pour les filles est bien la résultante d'un morphotype spécifique favorisant ou limitant la réalisation de bonnes performance chez les sprinters. Dans une prochaine étude plus approfondie, la prise en compte de l'âge physiologique donné par le stade pubertaire sera retenue car ce critère pubertaire et le somatotype de façon conjointe, pourraient avoir une influence prépondérante sur la performance sportive. La corrélation entre l'âge pubertaire et les résultats aux tests physiques a déjà été prouvé dans certaines études (Pineau, 1987 ; Belkacem, 2006).

\section{Remerciements}

Nous tenons à remercie :

$>\quad$ L'Institut National de la Jeunesse et des sport (INJS) Abidjan, pour sa collaboration au niveau de l'organisation du processus de recueil des données.

\section{References:}

Platonov, V. N., \& Jonco, N. (1988). L'entraînement sportif: théorie et méthodologie. Revue EPS.

Sedeaud, A., Marc, A., Marck, A., Dor, F., Schipman, J., Dorsey, M. \& Toussaint, J. F. (2014). BMI, a performance parameter for speed improvement. PloS one, 9(2), e90183.

MIMOUNI, N., \& ANTIPOV, E (1986). Profil morpho-fonctionnel des sportifs de haut niveau. Bulletin de l'ISTS.

Smirniotou, A., Katsikas, C., Paradisis, G., Argeitaki, P., Zacharogiannis, E., \& Tziortzis, S. (2008). Strength-power parameters as predictors of sprinting performance. Journal of Sports Medicine and Physical Fitness, 48(4), 447.

Ripari P., Di Blasio A., Di Iorio A., Albanese R., D’Anastasio R., \& Capasso L. (2008) : Somatotype and performance in a sedentary group of young people. Medicina dello Sport, 61(3): 357-63.

Yang, L. T., Wang, N., Li, Z. X., Liu, C., He, X., Zhang, J. F. \& Xi, H. J. (2015). Study on the adult physique with the Heath-Carter anthropometric somatotype in the Han of Xi'an, China. Anatomical science international, 91(2):180-7.

Larem A. et Pigearias B. (1990): Evaluation de la croissance en hauteur du corps de l'enfant ivoirien abidjanais age de 8 à 12 ans. Médecine d'Afrique Noire, 37 (12)

Carter, J. L., \& Heath, B. H. (1990). Somatotyping: development and applications (Vol. 5). Cambridge University Press. 
Anup, A., Nahida, P., Islam, R. N., \& Kitab, A. (2014). Importance of Anthropometric Characteristics in Athletic Performance from the Perspective of Bangladeshi National Level Athletes' Performance and Body Type. American Journal of Sports Science and Medicine, 2(4), 123-127.

Perroni, F., Vetrano, M., Camolese, G., Guidetti, L., \& Baldari, C. (2015). Anthropometric and Somatotype Characteristics of Young Soccer Players: Differences Among Categories, Subcategories, and Playing Position. The Journal of Strength \& Conditioning Research, 29(8), 2097-2104.

Vallois H.V. (1944) Les races humaines. Presse Universitaire de France ed. Paris, Que sais-je? 9ème édition 1976, 128.

Leone, M., \& Lariviere, G. (1996). Profil anthropometrique et biomoteur d'athletes adolescents soumis a un entrainement intensif. STAPS. Sciences et techniques des activités physiques et sportives, (41), 25-39.

Adodo, S. M., \& Agwubike, E. O. (2015). Somatotype of Nigerian male youth handball players in different playing positions. European Scientific Journal, 11(11): 213-221.

Vučetić, V., R Matković, B., \& Šentija, D. (2008). Morphological differences of elite Croatian track-and-field athletes. Collegium antropologicum, 32(3), 863-868.

Lowrey, G. H. (1986). Growth and development of children. chap. 4, p. 77121. Year Book Médical Publishers, Inc. Chicago. London.

Shepard, R. J. (1982). Physical activity and growth. chap.2, p.1-41. Year Book Medical Publishers, Inc. Chicago-London

Pineau, J. C. (1987). Importance de la puberté sur les résultats aux tests physiques chez les jeunes sportifs garçons et filles. Cahiers d'anthropologie et biométrie humaine, 5(1-2), 91-111.

Belkacem, K. (2006). L'aptitude aérobie des enfants durant le développement pubertaire selon le sexe. Thèse en ligne, disponible sur http://www.umc.edu.dz/buc/theses/sport/KHI4554.pdf. Consulté le 04/01/15. 